\title{
Review on Organ Preserving Techniques in Patients with Urethral Carcinoma
}

\author{
Mahmoud I Khalil ${ }^{1}$, Mohamed Gamal ${ }^{2}$ and Mohamed H Kamel ${ }^{1 *}$ \\ ${ }^{1}$ Department of Urology, University of Arkansas for Medical Sciences (UAMS), Little Rock, AR, USA \\ ${ }^{2}$ Department of Urology, Ain shams University, Cairo, Egypt
}

*Corresponding author: Mohamed H Kamel, M. D., F.A.C.S, Associate Professor, Department of Urology, University of Arkansas for Medical Sciences, 4301 West Markham \#540, Little Rock, AR 72205, USA.

Received Date: August 25, 2018

Published Date: September 12, 2018

\begin{abstract}
Tumors of the urethra are rare and often require radical surgery when dealing with locally advanced tumors to achieve adequate local control of the disease. In males, these procedures include partial or total penectomy for distal urethral carcinoma or total penectomy with cystoprostatectomy for proximal urethral carcinoma. In females with locally advanced urethral carcinoma, anterior exenteration may be required for better oncological outcomes. Patients diagnosed with urethral carcinoma at earlier stage may be amenable for genital sparing surgery which offers preservation of body image and better quality of life. We sought to review the current available literature describing different techniques of surgical management in patients with primary urethral carcinoma and subsequent survival and oncological outcomes.
\end{abstract}

Keywords: Organ preserving; Penile preserving; Urethral carcinoma

Abbreviations: AC: Adenocarcinoma; CRT: Chemoradiation Therapy; PUC: Primary Urethral Carcinoma; SCC: Squamous Cell Carcinoma; SEER: Surveillance Epidemiology and End Results; TUR: Transurethral Resection

\section{Introduction}

Primary Urethral carcinomas (PUC) constitute a small percentage of all Urothelial tumors arising from the urinary tract and accounting for $<1 \%$ of all malignancies in Europe [1]. In a review of PUC in the Surveillance, Epidemiology and End Results (SEER) database, the age adjusted incidence rate was 4.3 per million in men and 1.5 per million in women. The peaked incidence is at age group $\geq 75$ year (7.6 per million) [2]. The predominant histologic type of PUC is Urothelial carcinoma (55\%), followed by Squamous cell carcinoma (SCC; $21.5 \%$ ) and Adenocarcinoma (AC; 16.4\%) [2]. Various predisposing factors for male PUC have been reported including urethral strictures, chronic irritation subsequent to intermittent catheterization/urethroplasty, sexually transmitted diseases and external beam irradiation [3]. Urethral diverticula and recurrent urinary tract infections are associated with PUC in female [4]. In a SEER analysis over a period of 29 years, 1075 men and 540 women patients with PUC were identified, with annual age adjusted incidence rate of 4.3 per million and 1.5 per million for males and females respectively [5]. In the same analysis, the median 5 and 10-year overall survival rates was $46 \%$ and $29 \%$ respectively. The cancer specific survival at 5 and 10 years was $68 \%$ and $60 \%$ respectively [5].

Clinical staging, size and location of the urethral lesion are the important determinants in the management options and prognosis of patients with PUC [6]. In the proximal urethra, tumors tend to be more advanced and have a poor outcomes with 5 year diseasefree survival of 20-30\% with radical surgery alone [7]. By contrast, distal tumors (penile and glanular) tend to be of low stage and have better outcomes with cure rates of $70 \%-90 \%$ after treatment [8]. The prior goal for the urologist is to achieve local control for patients with PUC by surgical excision. Subsequently, the standard approach for an invasive disease in males has been partial or total penectomy for distal urethral tumors, and total penectomy with cystoprosatectomy for proximal tumors [9]. While in females, total urethrectomy up to anterior exenteration has been traditionally performed in invasive tumors [10]. Recently, urethra-preserving surgery is starting to be applicable in selected patients primarily for the distal urethral tumors [11]. 


\section{Methods}

A search strategy was performed to identify all published articles in peer-reviewed journals which describe different urethral preserving surgical techniques for patients with PUC from 1985 to November 2016. The search was conducted through PubMed, Web of Science and Cochrane Library Central Search. The search strategy included the following terms: "genital preservation", "penile preserving surgery", "organ sparing surgery", "urethral carcinoma" and "invasive carcinoma of the urethra". We considered English language publications not classified as case report, editorial, comment, letter or news. All pertinent studies collected were examined to extract relevant data pertained to conservative surgical therapy for PUC patients.

\section{Treatment of localized distal urethral carcinoma in male patients}

Smith et al. [12] described different operative techniques in his 18-Patient series of urethral cancer. Patients included in the study were reported to have anterior PUC with varying stage (T1-3) and grade (G1-3). Six out of the 18 patients had lymph node positive disease. In urethral lesions visible at the urethral meatus, slitting of the ventral surface with biopsy of the lesion and application of topical chemotherapy (5\% 5-fluorouracil) was done. The glandular urethra remained hypospadiac for at least 6 months to ensure local control. For confirmed $\mathrm{T} 1$ lesions, distal urethrectomy via bivalving and excision with 5-mm clear margin was done and then covered by buccal mucosal graft. The graft was quilted to the glanular spongiosum by $5 / 0$ absorbable sutures with urethral catheter for 5 days. A second stage urethral closure over a urethral catheter performed at least 3 months later

In the same study, Tumors extended into the glans was managed by glansectomy and hypospadias formation after excision of the urethra $5 \mathrm{~mm}$ proximal to the tumor. The corporal head is then covered with partial thickness skin graft harvested from the thigh. Distal corpectomy was done in patients who had tumors invading the distal corpora. In this study, no local recurrences reported over a mean follow up period of 26 months (12).

\section{Treatment of localized urethral carcinoma in female patients}

In female, local control is crucial to hinder local and systemic progression. In a long-term series of 27 female patients who had partial urethrectomy for PUC pT1-3, local recurrence with no bladder recurrence was reported in $6(22 \%)$ patients [13]. In those who recurred, the 5 years post-recurrence mortality rate was $71 \%$. More conservative management such as transurethral resection and laser ablation of small distal tumors demonstrate local failure rate of $16 \%$, with a cancer specific survival rate of $50 \%$ [13]. Radiotherapy might be an alternative to conserving surgery for PUC female patients. Garden's et al reported on 97 women with PUC treated by radiation. The reported 5-year local control rate was $64 \%$ with 7 -year cancer specific survival of $49 \%$. Patients with locally advanced tumors, tumors involved the entire urethra or fixed primary lesion were associated with poorer survival $(p<0.05)[14]$. Patients in this study received either external beam irradiation, brachytherapy or both (median, 65 Gy). Reported complications after radiotherapy includes urethral stenosis, fistula, cystitis and/ or hemorrhage.

\section{Treatment of advanced urethral carcinoma}

Local tumor control can be achieved by cisplatin-based chemotherapy in advanced urethral carcinomas. Multidisciplinary approach comprising of chemotherapy and surgery is essential in managing locally advanced PUC for better oncological and survival outcomes. In the retrospective study of Dayyani et al. [15], 44 patients with advanced PUC received neoadjuvant cisplatin-based chemotherapeutic regimens according to the underlying histology. The most prevalent histological types were squamous cell carcinoma and adenocarcinoma. The local response rate to various regimens was $72 \%$ with median overall survival of 32 months for the entire group of patients. Better survival data reported in patients who received salvage surgery $(\mathrm{n}=27)$ compared with those treated with chemotherapy alone (46.9 months vs. 21.7 months). Urethrectomy and radical cystectomy were the most performed procedures in this cohort. For locally advanced SCC of the urethra, local radiotherapy with concurrent radio sensitizing chemotherapeutic agents with curative intent had been described in various case serious and case reports [16-19]. Cohen et al. [20] reported survival outcomes of 18 patients who had invasive urethral carcinoma and received chemoradiation therapy (CRT) protocol. Tumor histology was SCC in 17 of them. Patients were subjected to External beam radiation therapy concurrently with chemotherapy (Mitomycin-C and 5-fluorouracil). Complete response was achieved in $83 \%$ of the cohort. The 5-year overall survival and disease-specific survival rates were $60 \%$ and $83 \%$ respectively. In all patients with no recurrence after CRT protocol $(n=10)$ had subsequent urethral stricture disease and required endoscopic and surgical management. The 5-year disease free survival rate was higher in patients received salvage surgery than patients received CRT only (72\% vs $54 \%)$.

\section{Treatment of urothelial carcinoma of the prostate}

Majority of patients diagnosed with noninvasive urothelial carcinoma of the prostate (Ta, Tis) have associated bladder urothelial tumors. Urethra-sparing approach with transurethral resection (TUR) and subsequent intravesical BCG instillation has been proven to be effective $[21,22]$. Follow up prostatic urethral biopsies is warranted to detect early tumor recurrence which is usually an indication for cystectomy. However, in patients with prostatic ductal involvement, better oncological results reported in patients underwent radical cystoprosatectomy rather than urethral/bladder conserving protocol. Local disease control rate of $57 \%-75 \%$ was reported in patients with ductal involvement received BCG instillation [22-24]. Prostatic stromal invasion is an indication for more aggressive treatment rather organ preservation, since the reported survival outcomes were dismal. In one report, the 5-year survival outcomes for patients with prostatic stromal invasion was $16 \%$ despite receiving radical treatment in $88 \%$. In other study of 24 patients with prostatic stromal invasion underwent radical cystoprosatectomy, 12 (50\%) were found to have positive lymph node positive disease with tendency to be above the iliac bifurcation [25]. 


\section{Conclusion}

Urethral conserving surgery in localized PUC is an alternative treatment option in both genders. In male patients, organ preserving approaches are hypospadias formation with topical chemotherapy, distal urethrectomy, glansectomy and distal corpectomy. In female patients, radiotherapy might be considered as alternative to surgery. In advanced urethral carcinoma, management should be based on multidisciplinary approach of urologists and oncologists. For noninvasive prostatic urethral carcinoma, local control is feasible by conservative treatment in the form of TUR and subsequent BCG instillation. However, radical treatment should be proposed in cases of local recurrence and extensive ductal or stromal involvement.

\section{References}

1. Gatta G, Van Der Zwan JM, Casali PG, Siesling S, Dei Tos AP, et al. (2011) Rare cancers are not so rare: The rare cancer burden in Europe. Eur J Cancer 47(17): 2493-2511.

2. Swartz MA, Porter MP, Lin DW, Weiss NS (2006) Incidence of primary urethral carcinoma in the United States. Urology 68(6): 1164-1168.

3. Hakenberg OW, Franke HJ, Froehner M, Wirth MP (2001) The treatment of primary urethral carcinoma--the dilemmas of a rare condition: experience with partial urethrectomy and adjuvant chemotherapy. Onkologie 24(1): 48-52.

4. Mohan H, Bal A, Punia RP, Bawa AS (2003) Squamous cell carcinoma of the prostate. International Journal of Urology 10(2): 114-116.

5. Libby B, Chao D, Schneider BF (2010) Non-surgical treatment of primary female urethral cancer. Rare tumors 2(3): e55.

6. Gakis G, Witjes JA, Comperat E, Cowan NC, De Santis M, et al. (2013) EAU guidelines on primary urethral carcinoma. European urology 64(5): 823-830.

7. Gheiler EL, Tefilli MV, Tiguert R, De Oliveira JG, Pontes JE, et al. (1998) Jr. Management of primary urethral cancer. Urology 52(3): 487-493.

8. Dinney CP, Johnson DE, Swanson DA, Babaian RJ, Von Eschenbach AC (1994) Therapy and prognosis for male anterior urethral carcinoma: an update. Urology 43(4): 506-514.

9. Zeidman EJ, Desmond P, Thompson IM (1992) Surgical treatment of carcinoma of the male urethra. The Urologic clinics of North America 19(2): 359-372.

10. Moinuddin Ali M, Klein FA, Hazra TA (1988) Primary female urethral carcinoma: A retrospective comparison of different treatment techniques. Cancer 62(1): 54-57.

11. Chitale SV, Szemere JC, Burgess NA, Sethia KK, Ball RY, et al. (2001) Surgical technique for the conservative management of distal urethral melanoma. Br J Plast Surg 54(4): 361-363.
12. Smith Y, Hadway P, Ahmed S, Perry MJ, Corbishley CM et al. (2007) Penile-preserving surgery for male distal urethral carcinoma. BJU Int 100(1): 82-87.

13. Dimarco DS, Dimarco CS, Zincke H, Webb MJ, Bass SE, et al. (2004) Surgical treatment for local control of female urethral carcinoma. Urol Oncol 22(5): 404-409.

14. Garden AS, Zagars GK, Delclos L (1993) Primary carcinoma of the female urethra. Results of radiation therapy. Cancer 71(10): 3102-3108.

15. Dayyani F, Pettaway CA, Kamat AM, Munsell MF, Sircar K, et al. (2013) Retrospective analysis of survival outcomes and the role of cisplatinbased chemotherapy in patients with urethral carcinomas referred to medical oncologists. Urol Oncol 31(7): 1171-1177.

16. Johnson DW, Kessler JF, Ferrigni RG, Anderson JD (1989) Low dose combined chemotherapy/radiotherapy in the management of locally advanced urethral squamous cell carcinoma. J Urol 141(3): 615-616.

17. Lutz ST, Huang DT (1995) Combined chemoradiotherapy for locally advanced squamous cell carcinoma of the bulbomembranous urethra: a case report. J Urol 153(5): 1616-1618.

18. Nicholson S, Tsang D, Summerton D (2008) Aggressive combinedmodality therapy for squamous cell carcinoma of the female urethra. Nature clinical practice Urology 5: 574-577.

19. Shah AB, Kalra JK, Silber L, Molho L (1985) Squamous cell cancer of female urethra. Successful treatment with chemoradiotherapy. Urology 25(3): 284-286.

20. Cohen MS, Triaca V, Billmeyer B, Hanley RS, Girshovich L, et al. (2008) Coordinated chemoradiation therapy with genital preservation for the treatment of primary invasive carcinoma of the male urethra. J Urol 179(2): 536-541.

21. Taylor JH, Davis J, Schellhammer P (2007) Long-term follow-up of intravesical bacillus Calmette-Guerin treatment for superficial transitional-cell carcinoma of the bladder involving the prostatic urethra. Clin Genitourin Cancer 5(6): 386-389.

22. Palou Redorta J, Schatteman P, Huguet Perez J, Segarra Tomas J, Rosales Bordes A, et al. (2006) Intravesical instillations with bacillus calmetteguerin for the treatment of carcinoma in situ involving prostatic ducts. Eur Urol 49(5): 834-838.

23. Palou J, Baniel J, Klotz L, Wood D, Cookson M, et al. (2007) Urothelial carcinoma of the prostate. Urology 69(1 Suppl): 50-61.

24. Hillyard RW, Ladaga L, Schellhammer PF (1988) Superficial transitional cell carcinoma of the bladder associated with mucosal involvement of the prostatic urethra: results of treatment with intravesical bacillus Calmette-Guerin. J Uro 139(2): 290-293.

25. Vazina A, Dugi D, Shariat SF, Evans J, Link R, et al. (2004) Stage specific lymph node metastasis mapping in radical cystectomy specimens. J Urol 171(5): 1830-1834. 